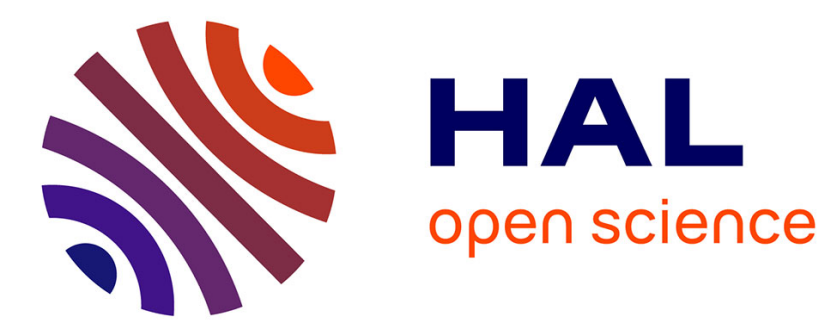

\title{
Rogue waves, rational solitons and wave turbulence theory
}

Bertrand Kibler, Kamal Hammani, Claire Michel, Christophe Finot, Antonio Picozzi

\section{- To cite this version:}

Bertrand Kibler, Kamal Hammani, Claire Michel, Christophe Finot, Antonio Picozzi. Rogue waves, rational solitons and wave turbulence theory. Physics Letters A, 2011, 375 (35), pp.3149-3155. 10.1016/j.physleta.2011.07.006 . hal-00607454

\section{HAL Id: hal-00607454 https://hal.science/hal-00607454}

Submitted on 8 Jul 2011

HAL is a multi-disciplinary open access archive for the deposit and dissemination of scientific research documents, whether they are published or not. The documents may come from teaching and research institutions in France or abroad, or from public or private research centers.
L'archive ouverte pluridisciplinaire HAL, est destinée au dépôt et à la diffusion de documents scientifiques de niveau recherche, publiés ou non, émanant des établissements d'enseignement et de recherche français ou étrangers, des laboratoires publics ou privés. 


\title{
Rogue waves, rational solitons
}

\section{and wave turbulence theory}

\author{
Bertrand Kibler,* Kamal Hammani, Claire Michel, Christophe Finot, and Antonio Picozzi \\ Laboratoire Interdisciplinaire Carnot de Bourgogne, UMR 5209 CNRS-Université de \\ Bourgogne, 9 Avenue Alain Savary, 21000 Dijon, France \\ *Corresponding author: Bertrand.Kibler@u-bourgogne.fr \\ Phone: +33 (0)3 80395932 - Fax: +33 (0)3 80395971
}

\begin{abstract}
Considering a simple one dimensional nonlinear Schrödinger optical model, we study the existence of rogue wave events in the highly incoherent state of the system and compare them with the recently identified hierarchy of rational soliton solutions. We show that rogue waves can emerge in the genuine turbulent regime and that their coherent deterministic description provided by the rational soliton solutions is compatible with an accurate statistical description of the random wave provided by the wave turbulence theory. Furthermore, the simulations reveal that even in the weakly nonlinear regime, the nonlinearity can play a key role in the emergence of an individual rogue wave event in a turbulent environment.

Keywords: Nonlinear optics; Pulse propagation and temporal solitons; Coherence; Statistical optics; Optical turbulence
\end{abstract}




\section{Introduction}

The evolution of random nonlinear waves can be characterized by the spontaneous emergence of short lived high amplitude waves. These rogue waves events that "appear from nowhere and disappear without a trace" [1] are among the most studied phenomena in nature in these last years [2]. Besides the hydrodynamic context [3-4], rogue waves have been recently identified in various different fields, including optical waves [5-7], capillary waves [8], superfluid helium [9] atmosphere [10] or microwaves [11]. Recent optical and hydrodynamics studies suggest that rogue wave events can be interpreted in the light of exact analytical solutions of 1D integrable nonlinear wave equations, the so-called Akhmediev breathers (ABs) [1-4,12], or more specifically their limiting cases of infinite spatial and temporal periods, the rational soliton (RS) solutions [13-15]. Because of this key property of localization in both the spatial and temporal domains, the RSs may be viewed as a kind of 'rogue wave prototype' [16]. The hierarchy of RSs has been found, in particular, in the framework of the integrable 1D nonlinear Schrödinger equation (NLS) equation, which is known to find several important applications in the framework of nonlinear optical waves [17]. A remarkable property of RSs is that they are characterized by an increasing value of the central amplitude of the wave field, a feature that can be used to interpret the emergence of rogue wave events of higher amplitudes from a chaotic field [15]. The Peregrine soliton solution refers to the first order rational solution [18]. It may be considered as a 'robust solution', in the sense that it can be excited even from non-ideal initial perturbations of the wave amplitude. In particular, its space-time profile may be closely approached into the nonlinear stage of the modulational instability, a feature that has been recently exploited in the context of nonlinear fiber optics to report its first experimental observation [19-20]. 
RSs are exact analytical solutions of integrable wave equations, and for this reason they may be regarded as a coherent and deterministic approach to the understanding of rogue wave phenomena. As a matter of fact, however, rogue waves events are known to spontaneously emerge from an incoherent turbulent state of the system. It is thus of a fundamental importance to study whether RSs can emerge from a turbulent environment (i.e., in more realistic oceanic conditions) [21,22]. In this respect, it is interesting to note that RSs have been recently identified in the midst of a modulationally unstable chaotic field [12,23]. However, besides these preliminary numerical observations, the problem of the existence of RS in a truly turbulent wave system has not been considered so far. Important questions, such as, e.g., the impact of the amount of incoherence in the system on the generation of RSs have not yet been considered. This actually constitutes a difficult problem, since the description of the turbulent wave system necessarily requires a statistical approach, whereas RSs are inherently coherent deterministic structures.

The present Letter is aimed at providing some physical insight into this vast problematic. We consider the emergence of rogue waves and RSs in a genuine turbulent wave system that can be described by the Wave Turbulence (WT) theory [24-26]. This theory is known to provide an accurate statistical description of random nonlinear waves in their weakly nonlinear regime of interaction. We address here the following important issue: Is the coherent description of rogue waves provided by RSs consistent with the WT description of the random wave? For this purpose, we consider a rather simple, nearly integrable, NLS model in which rogue wave events have been recently identified in the context of optics [27-28]. In particular, we recently showed that the amount of incoherence in the system has a deep impact on the properties of rogue waves, which may exhibit either intermittent or sporadic behaviors depending of the system's energy 
[28]. We showed that, by increasing the incoherence in the system, a transition occurs from the purely coherent and deterministic (quasi-)soliton regime toward the fully incoherent turbulent regime. From a different perspective, this last turbulent regime has been accurately described by using the WT theory [29-30], which thus brings the question of the existence of rogue wave events in the framework of the WT regime of the incoherent wave. We note in this respect that caution should be exercised when drawing conclusions as regard the applicability of the WT theory. It is indeed well-known that the existence of a permanent large scale coherent structure (such as a stable soliton or a condensate) leads to a breakdown of the WT theory [26,31]. However, contrary to solitons, rogue waves are, by nature, very short and very rare events. In this way, although the WT theory is inherently unable to describe rogue waves, their occurrence does not invalidate the applicability of the WT theory. We show that rogue waves can emerge in the genuine turbulent state of the random field and that their coherent deterministic description provided by RSs is compatible with an accurate WT description of the random waves. In particular, the nearly Gaussian statistics for the field amplitudes inherent to the WT theory is compatible with the asymmetric long tail observed in the pdf of the maxima of the field intensity. Furthermore, the simulations reveal that even in the weakly nonlinear regime, the nonlinearity can play a key role in the emergence of an individual rogue wave event. In this way we report a readily accessible optical setting in which the formation of spatiotemporal localized RS structures can be observed and studied in the WT regime of interaction.

\section{NLS model}

We consider the one-dimensional NLS equation in the presence of third-order dispersion (TOD),

$$
i \frac{\partial u}{\partial z}=-\frac{1}{2} \frac{\partial^{2} u}{\partial t^{2}}+i \sigma \frac{\partial^{3} u}{\partial t^{3}}-|u|^{2} u
$$


This equation is known to provide an accurate description of light propagation in optical fibers near by the zero-dispersion wavelength [17]. In writing Eq.(1), we normalized the problem with respect to the nonlinear length $\mathrm{L}_{0}=1 / \gamma \mathrm{P}$ and time $\tau_{0}=\left(\beta_{2} \mathrm{~L}_{0}\right)^{1 / 2}$, where $\gamma$ is the nonlinear coefficient, $\mathrm{P}$ the average power of the field and $\beta_{2}$ the second-order dispersion coefficient. In these units, the normalized TOD coefficient reads $\sigma=\beta_{3} /\left(6 \mathrm{~L}_{0}^{1 / 2} \beta_{2}^{3 / 2}\right), \beta_{3}$ being the TOD coefficient. The NLS Eq.(1) is Hamiltonian, $H=H_{L}+H_{N L}$, where $H_{L}=\int k(\omega)|u(\omega)|^{2} d \omega$ is the linear dispersive contribution and $\mathrm{H}_{\mathrm{NL}}=-1 / 2 \int|\mathrm{u}(\mathrm{t})|^{4} \mathrm{dt}$ the nonlinear contribution, $\mathrm{k}(\omega)=$ $\omega^{2} / 2+\sigma \omega^{3}$ being the linear dispersion relation. Equation (1) also conserves the normalized power $\mathrm{N}=\int|\mathrm{u}(\mathrm{t})|^{2} \mathrm{dt}$ and the momentum $\mathrm{P}=\int \omega|\mathrm{u}(\omega)|^{2} \mathrm{~d} \omega$. The WT theory is usually justified in the weakly nonlinear regime, where linear dispersive effects dominate nonlinear effects, $\mathrm{H}_{\mathrm{NL}} / \mathrm{H}_{\mathrm{L}}$ « 1 [26,32]. Equation (1) is known to admit quasi-soliton solutions: the TOD term $(\sigma \neq 0)$ breaks the integrability of the NLS equation, and the standard soliton solution slowly loses its power through a process analogous to Cherenkov radiation [26,33].

In a recent work [28], we showed that the NLS Eq.(1) exhibits a process analogous to wave condensation [24,31,34-37]: by studying the long term evolution of the wave, we identified a transition from the quasi-soliton state at small energies $\mathrm{H}$, toward the fully incoherent WT state at high energies. For completeness, we reported in Fig. 1(a) the condensation curve published in [28]. It can be interpreted by recalling that the energy $\mathrm{H}$ provides a natural measure of the amount of incoherence in the system: at high energies the system is too incoherent to generate a coherent quasi-soliton structure. We recall that the condensation curve is obtained by varying the energy $\mathrm{H}$, while the power ('number of quasi-particles') of the field $\mathrm{N}$ is kept constant. As discussed in detail in Ref. [28], the condensation curve is characterized by three distinct regimes: 
(i) persistent and coherent rogue quasi-solitons at small energies $\mathrm{H}$, (ii) intermittent-like rogue quasi-solitons that appear and disappear erratically at intermediate energies $\mathrm{H}$, and (iii) sporadic rogue waves events that emerge from turbulent fluctuations as bursts of light at high energies. Accordingly, it is only in the regime (ii) and the purely WT regime (iii) that the spontaneous emergence of extreme events was reported. Typical space-time intensity patterns in which rogue wave events have been identified are illustrated below in Fig. 1(b-c).
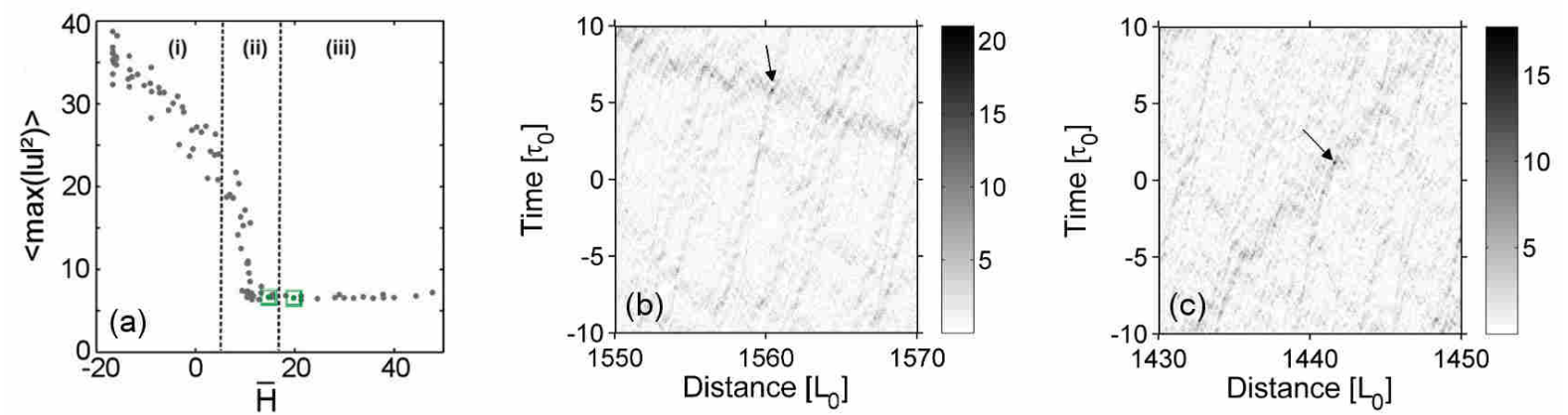

Figure 1: (a) Condensation-like curve: Average of the maximum intensity peak detected in the temporal window as a function of the Hamiltonian density $\bar{H}=H / T$, where $T$ is the size of the numerical temporal window ( $\sigma$ $=0.02)$. The power $\mathrm{N}$ of the wave is kept fixed $(\mathrm{N} / \mathrm{T}=1$ in normalized units). (b) Typical space-time intensity pattern showing an intermittent quasi-soliton propagating in the midst of turbulent fluctuations for $\overline{\mathrm{H}}=15.5$ [see the green square in regime (ii)]. (c) Typical space-time intensity pattern for $\overline{\mathrm{H}}=19.5$ showing the emergence of an extreme event at $\mathrm{z}=1441.6 \mathrm{~L}_{0}$ [see the green square in regime (iii)]. Black arrows indicate the rogue events studied in the following. 


\section{Temporal analysis and rational solitons}

In this section we investigate the temporal properties of the extreme events identified in the regimes (ii) and (iii) of the condensation curve [Fig. 1(a)]. We underline that several rogue wave events identified in regime (ii) and (iii) verify the standard hydrodynamic criterion [3], which defines a rogue wave as a wave that exhibits a maximal intensity higher than twice the average intensity among one third of the highest waves in the PDF of intensity maxima [28]. We start our analysis by considering the regime (ii), which is characterized by an intermittent-like behavior of the quasi-soliton structures, in the sense that it appears and disappears erratically during the propagation [28]. Because of its interaction with the turbulent fluctuations, the 'lifetime' of the quasi-soliton fluctuates significantly, so that the pdf of the intensity maxima exhibits an Lshaped distribution reminiscent of typical distributions characterizing extreme wave events [28].

\subsection{Filtering of rogue waves from fluctuations}

We identified several extreme events in the high intensity tail of the pdf distribution. A typical extreme event is reported in Fig. 2(a). It exhibits a maximum intensity of 22.9, which is much larger than 6.7, i.e., the value of the average of the maximum intensity detected in the long term evolution of the system (over $500 \mathrm{~L}_{0}$ ) [28]. In order to further analyze the properties of this extreme event, we calculated the corresponding spectrogram of the field at $\mathrm{z}=1560.4 \mathrm{~L}_{0}$ [see Fig. 2(b)]. The spectrogram constitutes a convenient tool for the identification of possible correlations between the spectral and temporal components of a field (see, e.g., [38]). Here, it reveals that the extreme event exhibits a high degree of localization in both the temporal and spectral domains. This allows us to accurately filter the extreme event from the surrounding turbulent fluctuations. The corresponding spectral and temporal filters used here are indicated in 
Fig. 2(b) by dashed lines. We underline that the temporal and frequency ranges of the filter do not affect our results in a significant way. Note that the spectral filter centered on the pump frequency also suppresses the dispersive wave radiated from the quasi-soliton, which is generated in the high-frequency edge of the spectrum (i.e., in the normal dispersion regime) [33]. This filtering procedure allows us to compare the structure of the extreme event with the analytical RS solutions of the NLS equation.
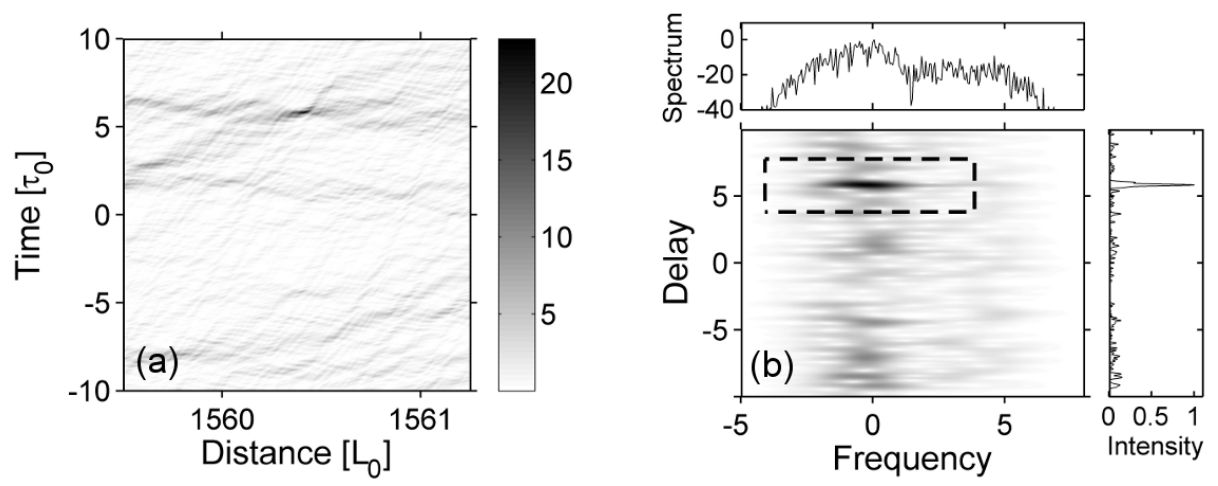

Figure 2: (a) Zoom on the space-time intensity pattern reported in Fig. 1(b) $[\overline{\mathrm{H}}=15.5$, regime (ii) $]$ showing the emergence of an extreme event at $\mathrm{z}=$ 1560.4 $\mathrm{L}_{0}$. (b) Numerical spectrogram of the optical field calculated at $\mathrm{z}=$ 1560.4 $\mathrm{L}_{0}$ revealing the spectro-temporal localization of the extreme structure (top window: spectrum profile in logarithmic scale, right window: temporal profile in linear scale). Dashed lines indicate the spectral and temporal filters used for subsequent analysis (see the text for details).

\subsection{Comparison with RS solutions}

The integrable NLS equation exhibits a large variety of analytical solutions. Among them one finds the hierarchy of RS solutions recently derived in Ref. [15] by using a modified Darboux 
transformation. The hierarchy of the solutions starts from the fundamental Peregrine soliton solution [18]. A remarkable property of RSs is that they are characterized by an increasing value of the central amplitude of the wave. RSs are described with the following basic structure characterized by the ratio of two polynomials $[15,39-40]: u_{j}=\left[(-1)^{j}+\left(G_{j}+i z H_{j}\right) / D_{j}\right] \exp (i z)$, where $G_{j}, H_{j}$ and $D_{j}$ are polynomials of both the spatial (z) and temporal (t) variables, where $j$ refers to the order of the solution. Note that explicit forms for the solutions, from the first-order to the fourth-order, have been given explicitly in Refs. [15,39]. Previous theoretical and numerical studies have also demonstrated that RSs are robust with respect to various types of perturbations $[23,41]$. Perturbed solutions that remain in the class of RSs have been also obtained by using an extended NLS equation with perturbing terms frequently encountered in the study of nonlinear optical waves. In particular, the role of TOD considered here has been analyzed in [41]. This study revealed that, for the small values of the TOD parameter considered here, the TOD term does not qualitatively affect the RS solution. Moreover, these solutions have been detected as the highest amplitude parts of a chaotic field by realizing simulations of the NLS equation starting from a continuous wave with superposed random fluctuations [12]. More generally, Calini and Schober have demonstrated that homoclinic orbits of the periodic solutions of the NLS equation persist in the perturbed system and some of their properties can be identified in the chaotic regime [42].

We now compare the extreme event filtered out from the turbulent fluctuations in Fig. 2(b) with different orders of analytic RSs. In order to compare them, the filtered field $u(t)$ is normalized in such a way that the power $\mathrm{N}$ contained in the reduced temporal window is the same as that of the analytical RS solution. Figure 3(a) reports the comparison between the corresponding extreme event detected in the simulation and the second order RS solution, which provided the best 
agreement with the simulations. The fact that the second order RS provides a better agreement with respect to the first and third orders RSs stems from the fact that its maximum intensity $\left(\mathrm{I}_{0}{ }^{\mathrm{RS}}\right.$ $=25)$ is closer to the maximum intensity of the field detected in the simulations $\left(\mathrm{I}_{0}{ }^{\text {num }}=22.9\right)$, while the intensity maxima of $\left|u_{1}\right|^{2}$ and $\left|u_{3}\right|^{2}$ are respectively 9 and 49. We remark in Fig. 3(a) that the intensity profile of the central pulse is in good agreement with the analytic RS solution, while the presence of turbulent fluctuations lead to an appreciable discrepancy in the side-lobes of the RS solution. Note in particular that a quantitative agreement between the numerics and the $R S$ is obtained for the value of the pulse peak (i.e., $\mathrm{I}_{0}{ }^{\mathrm{num}}=25.2, \mathrm{I}_{0}^{\mathrm{RS}}=25$ ). This good agreement is not due to the renormalization procedure, which only affects the integrated value of the wave amplitude over the numerical time window.

We also analyzed in our simulations an essential property of a rogue wave event, namely its degree of localization in the spatial and temporal domains [see the space-time profile in Fig. 3(b)]. We obtained a temporal FWHM of $\Delta \mathrm{t}^{\text {num }}=0.23 \tau_{0}$ and a spatial FWHM of $\Delta \mathrm{z}^{\text {num }}=0.23$ $\mathrm{L}_{0}$, which are in good agreement with the corresponding values of the analytical RS solution, $\Delta \mathrm{t}^{\mathrm{RS}}=0.3 \tau_{0}, \Delta \mathrm{z}^{\mathrm{RS}}=0.3 \mathrm{~L}_{0}$. Given the large amount of fluctuations surrounding the extreme even, we may consider this qualitative agreement as satisfactory. 

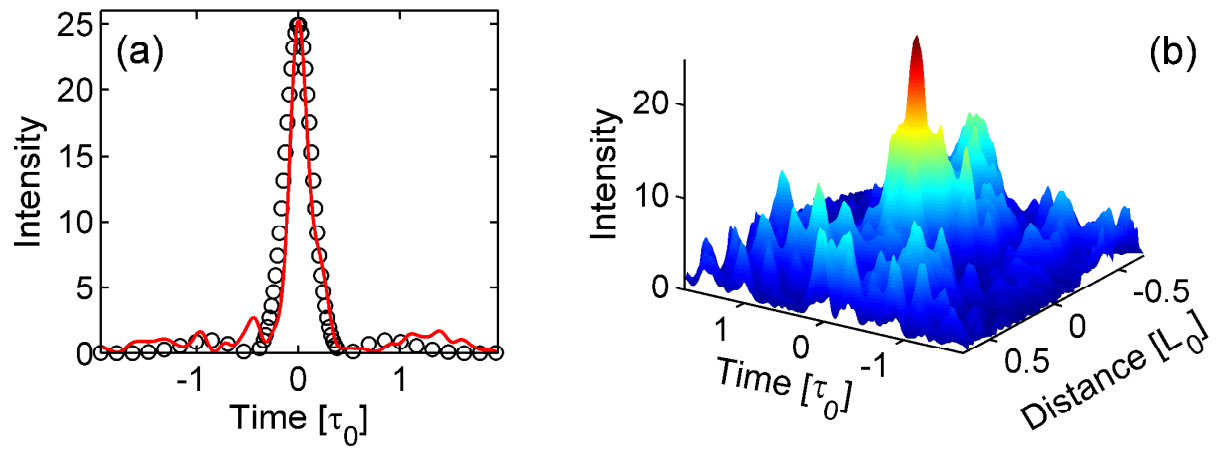

Figure 3: (a) Comparison of the filtered rogue wave event detected in regime (ii) in Fig. 2 (red solid line) with the second order analytical RS solution (black circles). (b) Spatio-temporal intensity profile of the filtered rogue wave event detected in the numerical simulations (see Fig. 2).

We have also performed this analysis for rogue wave events detected in the regime (iii), in which the system enters into the fully incoherent and weakly nonlinear regime. The spatio-temporal intensity pattern $|\mathrm{u}(\mathrm{z}, \mathrm{t})|^{2}$ reveals that very short-lived rogue wave events may still emerge from the turbulent field, although these extreme events become rare [see Fig. 1(c)]. In the space-time zoom pattern reported in Fig. 4(a), the extreme event exhibits a maximum intensity of $I_{0}{ }^{\text {num }}=$ 17.1. This value is lower than the previous one considered in regime (ii), but it is still much larger than 6.7 , which refers to the average of the maximum intensity detected in the long term evolution of the system [28]. Proceeding as in regime (ii), we report the spectrogram of the field at $\mathrm{z}=1441.6 \mathrm{~L}_{0}$ in Fig. 4(b). We remark that, in spite of the large amount of fluctuations inherent to the regime (iii), the extreme event can still be identified and is characterized by a high degree of localization in both the temporal and spectral domains. 
The space-time profile of the filtered field is compared with the second order analytical RS in Fig. 5(a). Globally, we may note that the agreement with the RS is poorer with respect to the previous analysis in regime (ii), as revealed by the comparison of Fig. 3(a) and Fig. 5(a). This observation has been confirmed through the analysis of several rogue wave events taken in regimes (ii) and (iii), and it can be simply interpreted as a consequence of the fact that the amount of turbulent fluctuations in regime (iii) is higher as compared to regime (ii). Note however in Fig. 5(a) that the central part of the pulse is still in good agreement with the analytical RS solution. In particular, the value of the peak pulse in the simulations $\mathrm{I}_{0}{ }^{\text {num }}=25.4$ is very close to the corresponding value of the $\mathrm{RS}, \mathrm{I}_{0}{ }^{\mathrm{RS}}=25$. Conversely, the significant discrepancies in the side-lobes can be ascribed to the large amount of incoherent fluctuations $(\overline{\mathrm{H}}$ 20) that characterizes the weakly nonlinear regime (iii). We also compared the temporal and spatial localization of the extreme event detected in the simulation with the corresponding localizations of the RS solution. We obtained at FWHM $\Delta \mathrm{t}^{\text {num }}=0.24 \tau_{0}$ and $\Delta \mathrm{z}^{\text {num }}=0.21 \mathrm{~L}_{0}$, which are still in rather good agreement with the corresponding RS's values $\left(\Delta \mathrm{t}^{\mathrm{RS}}=0.3 \tau_{0}, \Delta \mathrm{z}^{\mathrm{RS}}\right.$ $\left.=0.3 \mathrm{~L}_{0}\right)$.
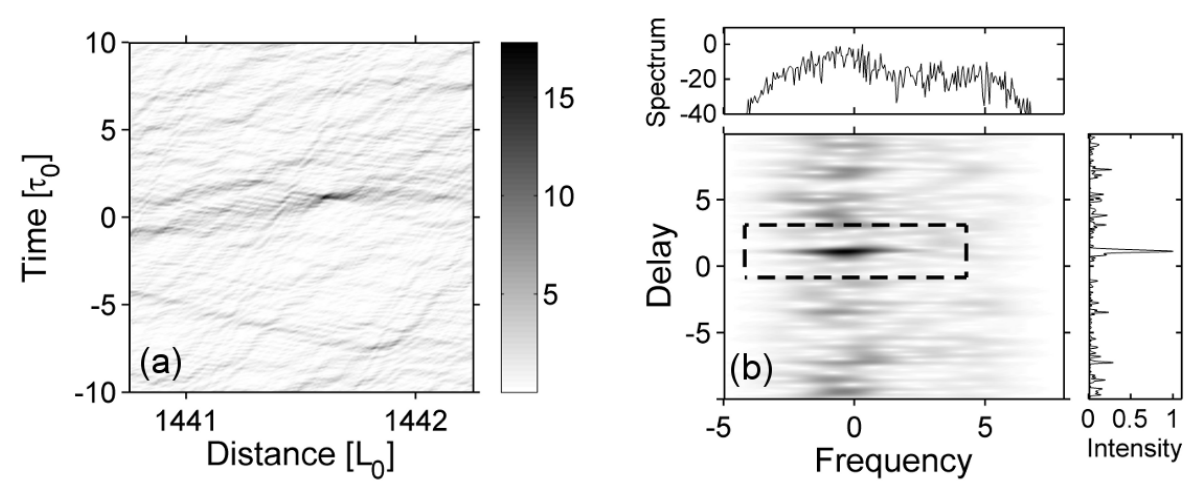

Figure 4: (a) Zoom on the space-time intensity pattern reported in Fig. 1(c) $[\overline{\mathrm{H}}=19.5$, regime (iii) $]$ showing the emergence of an extreme event at $\mathrm{z}=$ 1441.6 $\mathrm{L}_{0}$. (b) Numerical spectrogram of the optical field calculated at $\mathrm{z}=$ 
1441.6 $\mathrm{L}_{0}$ revealing the spectro-temporal localization of the extreme structure (top window: spectrum profile in logarithmic scale, right window: temporal profile in linear scale). Dashed lines indicate the spectral and temporal filters used for subsequent analysis (see the text for details).
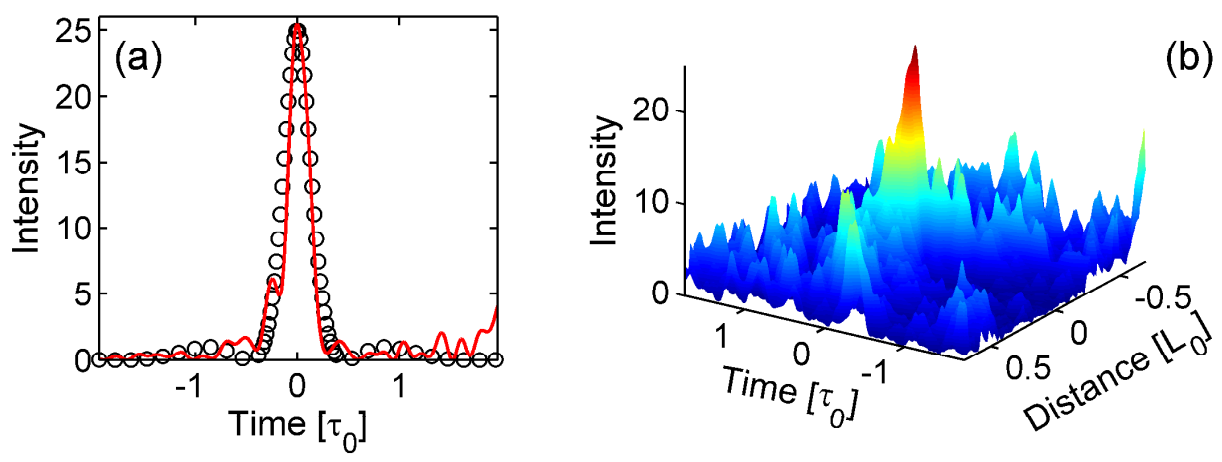

Figure 5: (a) Comparison of the filtered rogue wave event detected in regime (iii) in Fig. 4 (red solid line) with the third order analytical RS solution (black circles). (b) Spatio-temporal intensity profile of the filtered rogue wave event detected in the numerical simulations (see Fig. 4).

Finally note that, following the above filtering procedure, we analyzed several rogue wave events detected in both regimes (ii) and (iii), which confirmed the good agreement between their intensity profiles and the corresponding RS solutions. 


\section{Spectral analysis and wave turbulence theory}

In the framework of relatively small assumptions, the WT theory describes the spectral evolution of statistically homogeneous random waves in their weakly nonlinear regime of interaction, $\left|\mathrm{H}_{\mathrm{NL}} / \mathrm{H}_{\mathrm{L}}\right| \ll 1$ [43-45]. This theory has been essentially developed with the aim of describing fully developed turbulence in a dissipative system driven far from equilibrium by an external source [24-26], as it occurs, e.g., in some laser systems [46]. However, the kinetic equations also describe the nonequilibrium evolution of random nonlinear waves in a conservative and reversible (Hamiltonian) system. In analogy with kinetic gas theory, a closed system of incoherent nonlinear waves is expected to exhibit a thermalization process, which is characterized by an irreversible evolution of the system towards a thermodynamic equilibrium state. The WT theory describes the essential properties of this irreversible evolution towards the Rayleigh-Jeans equilibrium distribution [25]. This thermalization process has been studied in the framework of NLS-like equations in various different optical configurations [47], including supercontinuum generation [48-49], spontaneous repolarization [50], discrete systems [51], as well as in various optical media characterized by different nonlinearities [52-54].

In the WT theory the system evolves in the weakly nonlinear regime $\left(\left|\mathrm{H}_{\mathrm{NL}} / \mathrm{H}_{\mathrm{L}}\right|\right.$ «1), so that linear dispersive effects dominate the interaction and bring the random wave amplitude $\mathrm{u}(\mathrm{z}, \mathrm{t})$ close to a state of Gaussian statistics. This is in apparent contradiction with the intuitive idea that extreme wave events are usually associated to a deviation from Gaussian statistics. Actually, as reported in our previous work [28], the field amplitude $u(z, t)$ can exhibit a Gaussian statistics, while the existence of extreme events manifests itself through an asymmetric long tail distribution in the pdf of the intensity maxima. Our aim in this section is to show that the rogue wave events 
analyzed in the previous section can emerge from turbulent fluctuations whose spectral evolution is accurately described by WT theory.

The long term evolution of the NLS Eq.(1) has been recently shown to exhibit a process of anomalous thermalization [29-30]. It is characterized by an irreversible evolution of the field toward an equilibrium state of a fundamental different nature than the usual Rayleigh-Jeans distribution. Below we briefly sketch the essential properties of this anomalous thermalization and refer the reader to Refs. [29-30] for more technical details. In substance, when applied to the NLS Eq.(1), the WT theory reveals the existence of a local invariant in frequency space, $J(\omega)=n(\omega, z)-n(q-\omega, z)$, where $n(\omega, z)=\left\langle|\tilde{\mathrm{u}}|^{2}(\omega, \mathrm{z})\right\rangle$ is the averaged spectrum of the field amplitude, and $\mathrm{q}=-1 / 3 \sigma$. The invariant $J(\omega)$ is 'local' in the sense that it is verified for each individual frequency $\omega$ in frequency space. This local invariant originates in the following degenerate resonance of the system: the frequencies $(\omega, q-\omega)$ resonate with any pair of frequencies $\left(\omega^{\prime}, \mathrm{q}-\omega^{\prime}\right)$ because $\mathrm{k}(\omega)+\mathrm{k}(\mathrm{q}-\omega)=\mathrm{q}^{2} / 6$. Exploiting the existence of the invariant $J(\omega)$, one obtains the following WT kinetic equation governing the evolution of the averaged spectrum of the field:

$$
\partial_{\mathrm{z}} \mathrm{n}(\omega, \mathrm{z})=\frac{2}{3 \pi|\sigma|} \int \frac{F[n, J]}{\left|\omega-\omega_{1}\right|\left|\omega+\omega_{1}-q\right|} d \omega_{1}
$$

with the functional $F[n, J]=n_{\omega} n_{\omega_{1}}\left(n_{\omega}-J_{\omega}\right)\left(n_{\omega_{1}}-J_{\omega_{1}}\right)\left\{n_{\omega}^{-1}+\left(n_{\omega}-J_{\omega}\right)^{-1}-n_{\omega_{1}}^{-1}-\left(n_{\omega_{1}}-J_{\omega_{1}}\right)^{-1}\right\}$, where ' $\mathrm{n}_{\omega}$ ' stands here for $n(\omega, z)$. This kinetic equation has been integrated numerically and its evolution has been compared with the simulation of the NLS Eq.(1). We considered here both regimes (ii \& iii) of interaction previously analyzed for the following values of $\overline{\mathrm{H}}=15.5$ and $\overline{\mathrm{H}}=19.5$. The initial condition in the NLS Eq.(1) is a Gaussian spectrum with random spectral phases. The 
same Gaussian spectrum has been considered as initial condition in the kinetic Eq.(2). We report in Fig. 6 the NLS spectrum (red dashed line) and the corresponding kinetic spectrum (blue solid line) at the propagation distance in which the rogue wave events have been identified. We remark that a quantitative agreement is obtained between the NLS simulation and the kinetic simulation, without using any adjustable parameter.

It is interesting to note that the existence of the rogue wave event leads to a deviation between the NLS spectrum and the WT prediction in the very far tail of the spectrum (at $-80 \mathrm{~dB}$ ). This is illustrated in Fig. 6(a), which reports the NLS spectra just before (gray), during (red), and just after (green) the excitation of the rogue wave. The small deviation is only observed during a very short propagation distance, typically less than one $\mathrm{L}_{0}$. As will be discussed in the next Sec. 5 [see Fig. 7(b)], this small effect is a spectral manifestation of the rogue wave event, which, by nature, cannot be captured by the WT theory.

We finally note that, in its asymptotic evolution, the spectrum of the field tends to relax toward a 'local equilibrium state' of a different nature than the thermodynamic equilibrium state. In particular, it violates the property of energy equipartition: because of the existence of the local invariant $\mathrm{J}(\omega)$, the local equilibrium spectrum exhibits a highly asymmetric shape superimposed on a constant spectral pedestal [30]. 

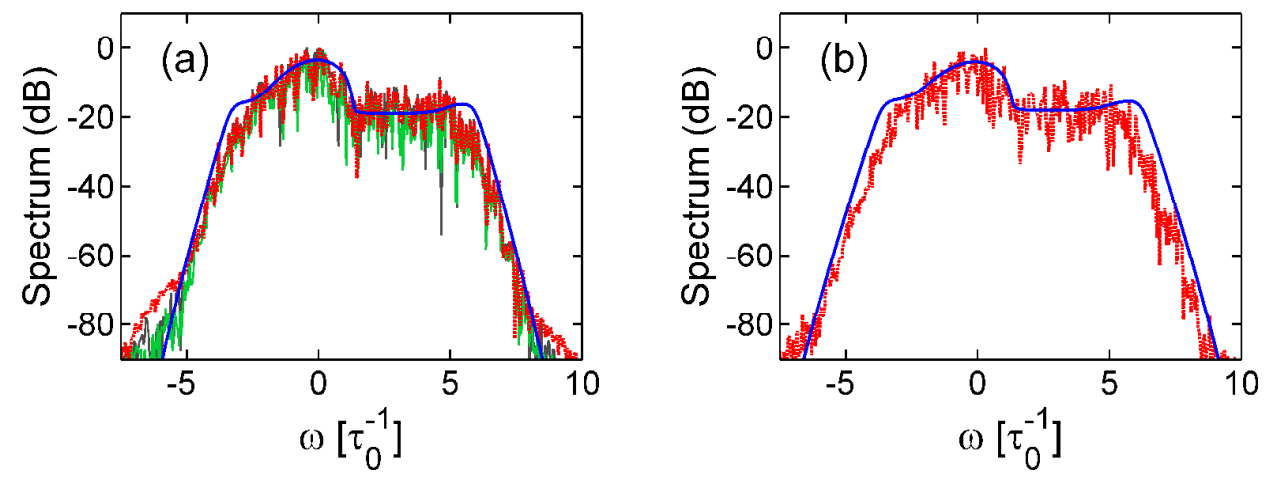

Figure 6: (a) Spectrum (in 10x $\log _{10}$-scale) obtained by integrating the NLS Eq.(1) at $\mathrm{z}=1560.4 \mathrm{~L}_{0}$ for $\overline{\mathrm{H}}=15.5$ in regime (ii) (red dashed line), compared with the spectrum obtained by integrating the WT kinetic Eq.(2) (blue solid line). Spectra obtained at $\mathrm{z}=1559.9 \mathrm{~L}_{0}$ (gray thin line) and $\mathrm{z}=1560.9 \mathrm{~L}_{0}$ (green thin line) are also reported. (b) Similar comparison at $\mathrm{z}=1441.6 \mathrm{~L}_{0}$ for $\overline{\mathrm{H}}=19.5$ in regime (iii). A satisfactory agreement is obtained, without using any adjustable parameter.

\section{Influence of the nonlinearity}

In the previous section we have shown that, despite the emergence of rogue wave events, the spectral evolution of the incoherent wave has been found in quantitative agreement with the WT theory. We have also shown in Section 3 that some rogue wave events exhibit properties reminiscent of the RS solutions. In this Section we shall see that even if one considers the weakly nonlinear regime of interaction, the nonlinearity plays an important role in the dynamics of emergence of an individual rogue wave event.

To assess the influence of the nonlinearity on the formation of the extreme event, we compare the NLS simulation of the rogue wave event analyzed in Fig. 3 with an identical simulation in 
which the nonlinearity is switched off two nonlinear lengths before the occurrence of the extreme event $\left(-2 \mathrm{~L}_{0}\right)$. More specifically, the extreme event occurs at $\mathrm{z}=1560.4 \mathrm{~L}_{0}$ in Fig. 3, we thus started the same NLS simulation at $\mathrm{z}=1558.4 \mathrm{~L}_{0}$ by removing the nonlinearity $(\gamma=0)$. The results are reported in Fig. 7(a). Note that we realized here the same filtering and renormalization procedures as those discussed in Fig. 3. We clearly note that the nonlinearity plays an important role in the formation of the extreme event. In particular, contrary to the nonlinear regime (see Sec. 3), the value of the maximum wave amplitude reached in the linear regime $\left(I_{0}^{\text {lin }} \sim 11.7\right.$ before renormalization) no longer satisfy the standard rogue wave criterion used in the context of hydrodynamic waves [3]. Furthermore, the temporal and spatial localizations of the detected 'linear event' are $\Delta \mathrm{t}^{\text {num }}=0.29 \tau_{0}$ and $\Delta \mathrm{z}^{\text {num }}=0.1 \mathrm{~L}_{0}$ : These values deviate in a significant way from the corresponding RS's values. This reveals that the detected rogue wave event does not result from a simple superposition of linear waves.
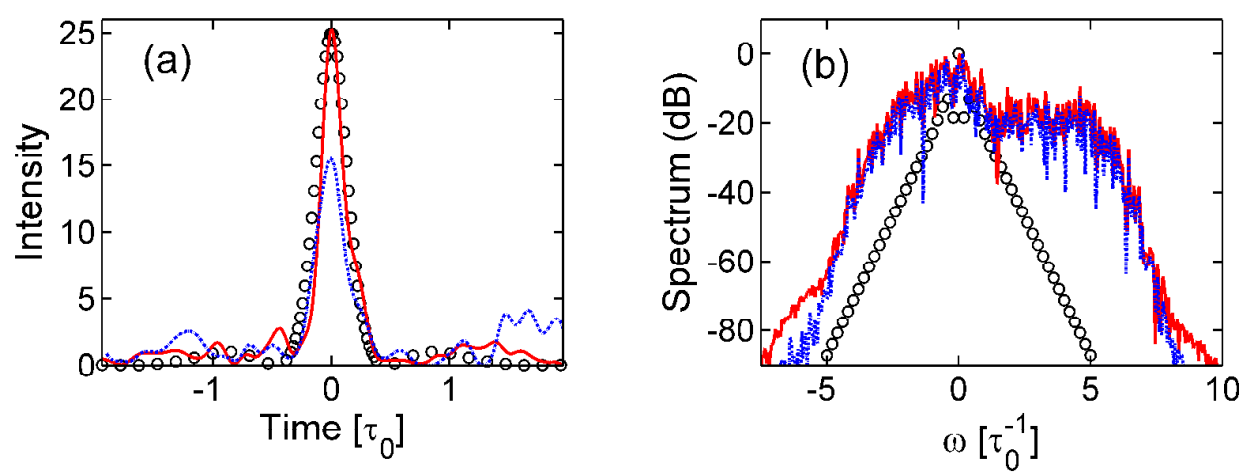

Figure 7: (a) Comparison of the rogue wave event analyzed previously in Figs. 3 (red solid line, nonlinear regime) with the same event obtained by simulating the NLS equation in the 'linear' regime, $\gamma=0$ (blue dashed line, see the text for more details). The plot of the second order RS solution is also shown (black circles). (b) Corresponding wave spectra (in $10 x \log _{10}$-scale) of the field in the nonlinear regime 
(red solid line) and in the "linear" regime (blue dashed line). Spectrum of the second order RS solution is also shown (black circles).

We also report in Fig. 7(b) the corresponding numerical spectra of the fields in the linear and nonlinear regimes considered in Fig. 7(a). Note that these spectra do not account for the filtering procedure discussed in Sec. 3. We can remark that both spectra are almost indistinguishable, so that they cannot be used to detect easily the existence of the rogue wave event. This point deserves to be discussed in relation with the fact that the Peregrine soliton and RSs are known to be characterized by a specific triangular-shaped spectrum that appears at an early stage of their evolution [55], a property that was confirmed experimentally [19-20]. For this reason, such triangular-shaped spectra were proposed as a warning signal for the emergence of extreme wave events in oceans $[55,56]$. The analysis reported here indicates that this suggestion is not relevant when the extreme wave event emerges from a turbulent environment in a perturbed-NLSE system. Indeed, the slight difference observed in the far tail of the spectrum for $\overline{\mathrm{H}}=15.5$ in regime (ii) becomes undetectable in the highly incoherent regime (iii), as illustrated in Fig. 6(b) for $\overline{\mathrm{H}}=19.5$.

\section{Conclusion}

In summary, through the analysis of the long term evolution of the wave system, we investigated the spectral and temporal properties of the incoherent field in its highly incoherent regime of interaction. We showed that intermittent-like rogue quasi-solitons [regime (ii)] and sporadic rogue wave events [regime (iii)], both exhibit properties reminiscent of the hierarchy of the RS solutions. This coherent and deterministic description of rogue waves coexists with the statistical 
description of the random field, which is found in quantitative agreement with the WT theory without using adjustable parameters. We also showed that the nonlinearity can play an important role in the formation of an individual rogue wave event, a feature that should deserve to be analyzed in further details, in relation with the recent work [57]. The complementary temporal and spectral analyses of the turbulent field and of the rogue wave events indicate that the localized RS coherent structures can be observed experimentally during the natural process of optical wave thermalization to equilibrium. It would be interesting to extend this work to the study of RSs in a forced-dissipative system driven far from thermal equilibrium by an external source $[25,58]$.

Acknowledgments: This research was supported by the Agence Nationale de la Recherche (COSTUME project, ANR-08-SYSC-004-03; MANUREVA project, ANR-08-SYSC019) and by the Conseil Régional de Bourgogne. 


\section{References}

1. N. Akhmediev, A. Ankiewicz, M. Taki, Phys. Lett. A 373 (2009) 675-678.

2. Special Issue: "Discussion \& Debate: Rogue Waves - Towars a Unifying Concept?" The European Physical Journal - Special Topics 185 (2010).

3. C. Kharif, E. Pelinovsky, A. Slunyaev, Rogue Waves in the Ocean, Springer-Verlag, Berlin, 2009.

4. A.R. Osborne, Nonlinear Ocean Waves and the Inverse Scattering Method, Academic Press, San Diego, 2010.

5. D. R. Solli, C. Ropers, P. Koonath, B. Jalali, Nature 450 (2007) 1054-1057.

6. A. Montina, U. Bortolozzo, S. Residori, F. T. Arecchi, Phys. Rev. Lett. 103 (2009) 173901.

7. J. Kasparian, P. Béjot, J. P. Wolf, J. M. Dudley, Opt. Express 17 (2009) 12070-12075.

8. M. Shats, H. Punzmann, H. Xia, Phys. Rev. Lett. 104 (2010) 104503.

9. A.N. Ganshin, V.B. Efimov, G.V. Kolmakov, L.P. Mezhov-Deglin, P.V. McClintock Phys. Rev. Lett. 101 (2008) 065303.

10. L. Stenflo, M. Marklund, J. Plasma Physics 76 (2010) 293-295.

11. R. Höhmann, U. Kuhl, H.-J. Stöckmann, L. Kaplan, E. J. Heller, Phys. Rev. Lett. 104 (2010) 093901.

12. N. Akhmediev, J.M. Soto-Crespo, A. Ankiewicz, Phys. Lett. A 373 (2009) 2137-2145.

13. K. L. Henderson, D. H. Peregrine, J. W. Dold, Wave Motion 29 (1999) 341-361.

14. K. B. Dysthe, K. Trulsen, Physica Scripta 82 (1999) 48-52.

15. N. Akhmediev, A. Ankiewicz, J.M. Soto-Crespo, Phys. Rev. E 80 (2009) 026601.

16. V.I. Shrira, V.V. Geoigjaev, J. Eng. Math. 67 (2010) 11-22. 
17. Y. S. Kivshar and G. P. Agrawal, Optical Solitons: From Fibers to Photonic Crystals, Academic Press, San Diego, 2003.

18. D.H. Peregrine, J. Aust. Math. Soc. Ser. B 25 (1983) 16-43.

19. B. Kibler, J. Fatome, C. Finot, G. Millot, F. Dias, G. Genty, N. Akhmediev, J.M. Dudley, Nat. Phys. 6 (2010) 790-795.

20. K. Hammani, B. Kibler, C. Finot, J. Fatome, J.M. Dudley, G. Millot, Opt. Lett. 36 (2011) $112-114$

21. M. Onorato, A. R. Osborne, M. Serio, S. Bertone, Phys. Rev. Lett. 86 (2001) 5831-5834.

22. M. Onorato, A. Osborne, M. Serio, L. Cavaleri, C. Brandini, C.T. Stansberg, Europ. Jour. of Mech. B/Fluids 25 (2006) 586.

23. A. Ankiewicz, J. M. Soto-Crespo, N. Akhmediev, Phys. Rev. E 81 (2010) 046602.

24. S. Dyachenko, A.C. Newell, A. Pushkarev, V.E. Zakharov, Physica D 57 (1992) 96-160.

25. V. Zakharov, V. L'vov, G. Falkovich, Kolmogorov Spectra of Turbulence I, Springer, Berlin, 1992.

26. V. Zakharov, F. Dias, A. Pushkarev, Phys. Reports 398 (2004) 1-65.

27. M. Taki, A. Mussot, A. Kudlinski, E. Louvergneaux, M. Kolobov, M. Douay, Phys. Lett. A 374 (2010) 691-695.

28. K. Hammani, B. Kibler, C. Finot, A. Picozzi, Phys. Lett. A 374 (2010) 3585-3589.

29. P. Suret, S. Randoux, H. Jauslin, A. Picozzi, Phys. Rev. Lett. 104 (2010) 054101.

30. C. Michel, P. Suret, S. Randoux, H.R. Jauslin, A. Picozzi, Opt. Lett. 35 (2010) 23672369.

31. G. Düring, A. Picozzi, S. Rica, Physica D 238 (2009) 1524-1549. 
32. An accurate description of the validity of the WT theory for Eq.(1) requires an improved criterion, for details see C. Michel, J. Garnier, P. Suret, S. Randoux, A. Picozzi, Lett. Math. Phys. 96 (2011) 415-447.

33. See, e.g., N. Akhmediev and M. Karlsson, Phys. Rev. A 51 (1995) 2602-2607.

34. M. J. Davis, S. A. Morgan, K. Burnett, Phys. Rev. Lett. 87 (2001) 160402.

35. B. Rumpf and A.C. Newell, Phys. Rev. Lett. 87 (2001) 054102.

36. C. Connaughton, C. Josserand, A. Picozzi, Y. Pomeau, S. Rica, Phys. Rev. Lett. 95 (2005) 263901.

37. P. Aschieri, J. Garnier, C. Michel, V. Doya, A. Picozzi, Phys. Rev. A 83 (2011) 033838.

38. J.M. Dudley, G. Genty, S. Coen, Rev. Mod. Phys. 78 (2006) 1135-1184.

39. A. Ankiewicz, P. A. Clarkson, N. Akhmediev, J. Phys. A: Math. Theor. 43 (2010) 122002.

40. P. Dubard, V. B. Matveev, Nat. Hazards Earth Syst. Sci. 11 (2011) 667-672.

41. A. Ankiewicz, N. Devine, N. Akhmediev, Phys. Lett. A 373 (2009) 3997-4000.

42. A. Calini, C.M. Schober, Phys. Lett. A 298 (2002) 335-349.

43. J. Benney and P. G. Saffman, Proc.R. Soc. London A 289 (1966) 301.

44. C. Newell, Rev. Geophys. 6 (1968) 1.

45. C. Newell, S. Nazarenko, L. Biven, Physica D 152 (2001) 520.

46. S.A. Babin, D.V. Churkin, A.E. Ismagulov, S.I. Kablukov, E.V. Podivilov, J. Opt. Soc. Am. B 24 (2007) 1729-1738.

47. A. Picozzi, Opt. Express 15 (2007) 9063-9083.

48. B. Barviau, B. Kibler, A. Kudlinski, A. Mussot, G. Millot, A. Picozzi, Opt. Express 17 (2009) 7392-7406. 
49. B. Barviau, B. Kibler, A. Picozzi, Phys. Rev. A 79 (2009) 063840.

50. A. Picozzi, Opt. Express 16 (2008) 17171-17185.

51. Y. Silberberg, Y. Lahini, Y. Bromberg, E. Small, R. Morandotti, Phys. Rev. Lett. 102 (2009) 233904.

52. U. Bortolozzo, J. Laurie, S. Nazarenko, S. Residori, J. Opt. Soc. Am. B 26 (2009) 22802284.

53. S. Lagrange, H.R. Jauslin, A. Picozzi, Europhys. Lett. 79 (2007) 64001.

54. A. Picozzi, P. Aschieri, Phys. Rev. E 72 (2005) 046606.

55. N. Akhmediev, A. Ankiewicz, J.M. Soto-Crespo, J.M. Dudley, Phys. Lett. A 375 (2011) $541-544$

56. N. Akhmediev, A. Ankiewicz, J.M. Soto-Crespo, J.M. Dudley, Phys. Lett. A 375 (2011) 775-779.

57. F. T. Arecchi, U. Bortolozzo, A. Montina, and S. Residori, Phys. Rev. Lett. 106 (2011) 153901.

58. B. Rumpf, A.C. Newell, V. Zakharov, Phys. Rev. Lett. 103 (2009) 074502. 